\title{
Competitive Equilibrium when Preferences Change over Time*
}

\author{
Erzo G.J. Luttmer ${ }^{\dagger} \quad$ Thomas Mariotti $^{\ddagger}$
}

June 24, 2002

\begin{abstract}
A bstract
We show the existence of a competitive equilibrium in an economy with many consumers whose preferences may change over time. The demand correspondence of an individual consumer is determined by the set of subgameperfect equilibrium outcomes in his or her intrapersonal game. For additively separable preferences with continuous period utility functions that are unbounded above, this demand correspondence will satisfy the usual boundary conditions. Whenever consumers can recall their own mixed actions, this correspondence is convex valued. This ensures the existence of a symmetric competitive equilibrium.
\end{abstract}

\section{Int roduction}

We show the existence of a competitive equilibrium in a dynamic exchange economy with many consumers whose preferences may change over time. At any given date, consumers are endowed with preferences over consumption sequences. As in Strotz (1956), these preferences may change as time evolves. Following Pollak (1968) and, more recently, Laibson (1997) and many others, consumption choices are determined by the outcome of an intrapersonal game played by successive incarnations of the same consumer. Consumers correctly anticipate how their future behavior is affected by changes in preferences. As pointed out by Peleg and Yaari (1973), such an intrapersonal game need not have a Markov perfect equilibrium. Following Goldman (1980), we therefore study subgame-perfect equilibria. In the most general setup we consider, the strategies in this game are allowed to include mixing, and past mixing

${ }^{*}$ This is work in progress. Comments welcome. We thank Michele Piccione for useful comments and suggestions.

${ }^{\dagger}$ Department of Economics, University of Minnesota, and CEPR.

${ }^{\ddagger}$ Department of Economics, London School of Economics, GREMAQ, Université de Toulouse, 1, and CEPR. 
behavior of a consumer is allowed to affect his current choices. As a result of this, the individual demand correspondences have convex values. This allows for a competitive equilibrium that is symmetric in the sense that consumers of the same type make the same (randomized) choices. Competitive equilibria also exist in the absence of observable mixed strategies, but individual demand correspondences are no longer convex, and the competitive equilibrium may have to be asymmetric.

One motivation for this paper is the recent work on modeling agents with timeinconsistent preferences (Laibson (1997), Harris and Laibson (2001)). In Luttmer and Mariotti (2000) we explicitly solve for the competitive equilibrium prices in an economy with consumers whose preferences are homothetic and additively separable with subjective discount factors that exhibit present bias. This present bias is shown to be a potential source of volatility in aggregate wealth. As in many applications, homotheticity makes the problem tractable. But without arguments that show that competitive equilibria can be properly defined for more general preferences, it is not clear that one can view homotheticity as a convenient approximation. This paper takes some steps towards filling this gap. In a companion paper we discuss in more detail some aspects of homothetic preferences that are special when preferences change over time (Luttmer and Mariotti (2002)).

The paper is organized as follows. In Section 2, we describe a general class of intrapersonal games, and characterize the set of subgame-perfect equilibrium outcomes with observable mixed strategies. Section 3 applies this result to derive the existence of a competitive equilibrium in a dynamic exchange economy with consumers whose tastes may change over time. Section 4 concludes.

\section{An Intrapersonal Game}

We describe a game that will be interpreted in Section 3 as the game played by the incarnations of a finitely-lived price-taking consumer who can trade a single consumption good across time.

\subsection{The Physical Environment}

There is a finite number of periods, indexed by $t=1, \ldots, T$. A non-empty complete separable metric space $H_{0}$ represents the set of starting points of the game. For each $t=1, \ldots, T$, the action of the date- $t$ consumer is chosen fom a non-empty complete separable metric space $C_{t}$. A closed subset $H_{t} \subset H_{0} \times{ }_{t=1} C_{s}$ represents the set of histories that can occur up to and including date $t$. The set of actions available to the date- $t$ consumer following any history in $H_{t-1}$ is given by a correspondence $A_{t}: H_{t-1}{ }^{3} C_{t}$ that is continuous and has non-empty and compact values. The sets of possible histories $H_{t}$ are defined recursively via $H_{t}=$ graph $A_{t}$ for $t=1, \ldots, T$. For any history $h_{t-1} \in H_{t-1}$, let $\Gamma_{t}\left(h_{t-1}\right)$ be the s\& of possible continuation histories following history $h_{t}$. It follows that $\Gamma_{t}: H_{t-1}{ }^{3}{ }_{s=t} C_{s}$ is a continuous correspondence 
with non-empty and compact values. The date- $t$ consumer has expected-utility preferences with a continuous von-Neumann-Morgenstern utility function $U_{t}: H_{T} \rightarrow \mathbf{R}$.

\subsection{Observable Mixed Strategies}

In an intrapersonal game, it is natural to assume that the mixed action chosen by the date- $t$ consumer, and not only the outcome of such a mixed action, can be observed by his successors. Observable mixed actions can be accounted for by defining extended histories. For any $t=1, \ldots, T$, the set of date- $t$ extended histories $\boldsymbol{P}_{t}$ is given by: ${ }^{1}$

$$
A_{t}=\left(h_{t}, m_{t}\right): h_{t} \in H_{t}, m_{t} \in \mathrm{Y}_{s=1}^{t} \Delta\left(A_{s}\left(\operatorname{proj}_{H_{s-1}} h_{t}\right)\right) .
$$

That is, a date- $t$ extended history specifies a physical history $h_{t}$ as well as a sequence $m_{t}$ of sequentially feasible mixed actions up to and including date $t$. One can verify that $\boldsymbol{A}_{t}$ is closed for all $t=1, \ldots, T$. For symmetry, we set $\boldsymbol{A}_{0}=H_{0}$ and interpret $m_{0}$ as an empty symbol. We can now define strategies.

Definition 1 For any $t=1, \ldots, T$, a strategy for the date $t$ consumer is a Borel measurable function $\gamma_{t}: A_{t-1} \rightarrow \Delta\left(C_{t}\right)$ that satisfies:

$$
\operatorname{supp} \gamma_{t}\left(\cdot \mid \boldsymbol{\ell}_{t-1}\right) \subset A_{t}\left(\operatorname{proj}_{H_{\mathrm{t}-1}} \boldsymbol{\otimes}_{t-1}\right)
$$

for all $\boldsymbol{\varphi}_{t-1} \in \AA_{t-1}$.

For any $h_{0} \in H_{0}$, a strategy combination $\gamma=\left(\gamma_{1}, \ldots, \gamma_{T}\right)$ induces a probability distribution over the set of possible histories in the subgame $h_{0}$. We refer to this as the path induced by $\gamma$ in the subgame $h_{0}$.

Definition 2 An intrapersonal equilibrium is a strategy combination $\gamma$ such that for all $t=1, \ldots, T$, and any history $\boldsymbol{\theta}_{t-1} \in \boldsymbol{R}_{t-1}$, the date- $t$ consumer cannot increase his utility in the subgame $\boldsymbol{\tau}_{t-1}$ by using a strategy other than $\gamma_{t}$.

Harris (1985) readily implies that an intrapersonal equilibrium exists: any subgameperfect equilibrium in pure strategies can be turned into an intrapersonal equilibrium. However, the subgame-perfect equilibrium correspondence may fail to be convex valued, even if mixed strategies are taken into account. To see this, consider the twoplayer game of perfect information illustrated in Figure 1. It is immediate that $(U, u)$ and $\left(D, d^{\prime}\right)$ are subgame-perfect equilibrium paths. For Player 1 to be indifferent between $U$ and $D$, it must be that Player 2 is mixing with equal probability between $u$ and $d$ following $U$. This means that there is no subgame-perfect equilibrium in which the equilibrium path is a lottery over $(U, u)$ and $\left(D, d^{\prime}\right)$ only. ${ }^{2}$

\footnotetext{
${ }^{1}$ The set of Borel probability measures over a complete separable metric space $\mathbf{X}$ is denoted by $\Delta(\mathrm{X})$, and is endowed with the usual weak* topology.

${ }^{2}$ This example shows that Proposition 36 in Harris, Reny and Robson (1995) is not correct as stated: the set of subgame-perfect equilibrium payoff vectors of this game is not convex.
} 


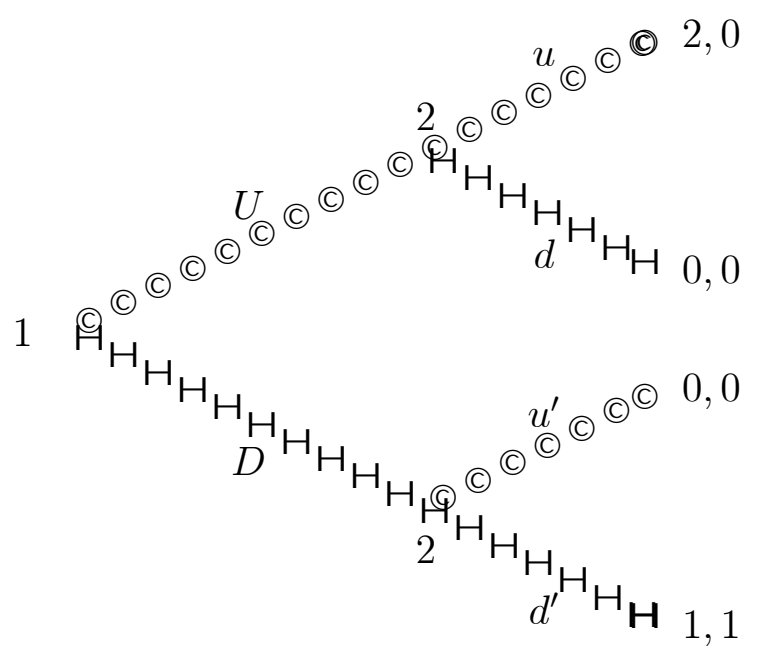

Figure 1

However, it is easy to implement any path in the convex hull of subgame-perfect equilibrium outcomes if Player 2 can observe the mixed action used by Player 1 . Consider for instance the path obtained by randomizing over $(U, u)$ and $\left(D, d^{\prime}\right)$ with equal probabilities. A subgame-perfect equilibrium with observable mixed strategies that implement this path is as follows. If Player 1 randomizes with equal probabilities between $U$ and $D$, then Player 2 chooses $u$ after $U$ and $d^{\prime}$ after $D$. For any other randomization by Player 1, Player 2 chooses $d$ after $U$ and $d^{\prime}$ after $D$. It is optimal for Player 1 to randomize in the prescribed way, for he obtains a payoff of $3 / 2$, while he would get at most 1 by deviating. The key difference with a subgame-perfect equilibrium without observable mixed strategies is that Player 1 is not indifferent between the two actions $U$ and $D$ in equilibrium.

\subsection{The Backward Program}

Fix some correspondence $D_{t+1}: H_{t}{ }^{3} \Delta\left(\Gamma_{t+1}(\cdot)\right)$ that describes equilibrium continuation paths following any history in $H_{t}$. This date- $t$ continuation correspondence is taken to be upper hemicontinuous and to have non-empty convex and compact values.

We will need to merge continuation paths in $D_{t+1}\left(H_{t}\right)$ with mixed actions of the date- $t$ consumer to obtain continuation paths for histories in $H_{t-1}$. At a minimum, the continuation paths following a history $h_{t-1} \in H_{t-1}$ must be in:

$$
\mathcal{P} D_{t+1}\left(h_{t-1}\right)=\left\{\mu: \mu=\nu \otimes \lambda, \nu \in \Delta\left(A_{t}\left(h_{t-1}\right)\right), \lambda\left(\cdot \mid c_{t}\right) \in D_{t+1}\left(h_{t-1}, c_{t}\right)\right\} .
$$

This is the set of all probability distributions on $\Gamma_{t}\left(h_{t-1}\right)$ that are consistent with marginals in $\Delta\left(A_{t}\left(h_{t-1}\right)\right)$ and conditionals that map $c_{t}$ into $D_{t+1}\left(h_{t-1}, c_{t}\right)$. 
Lemma $1 \mathcal{P} D_{t+1}: H_{t-1}{ }^{3} \quad \Delta\left(\Gamma_{t}(\cdot)\right)$ is an upper hemicontinuous correspondence with non-empty values that are convex and compact.

The proof of this lemma is implied by results in Mariotti (2000) who builds on earlier work by Simon and Zame (1990) and Harris, Reny and Robson (1995). The convexity of $\mathcal{P} D_{t+1}\left(h_{t-1}\right)$ follows from the assumed convexity of $D_{t+1}\left(h_{t}\right)$.

For any $h_{t} \in H_{t}$, the utility of the date- $t$ consumer from the worst possible continuation path in $D_{t+1}\left(h_{t}\right)$ is:

$$
M_{t}\left(h_{t}\right)=\min ^{1 / 2 Z} U_{t}\left(h_{t}, z\right) \mathrm{d} \mu(z): \mu \in D_{t+1}\left(h_{t}\right)^{3 / 4} .
$$

We let $\mathcal{A} M_{t}\left(h_{t}\right)$ denote the set of probabilities that attain this minimum. For any $h_{t-1} \in H_{t-1}$, the date- $t$ consumer can guarantee an approximate utility level of:

$$
V_{t}\left(h_{t-1}\right)=\sup \left\{M_{t}\left(h_{t-1}, c_{t}\right): c_{t} \in A_{t}\left(h_{t-1}\right)\right\} .
$$

Now define:

$$
\mathcal{B} D_{t+1}\left(h_{t-1}\right)={ }^{1 / 2} \mu \in \mathcal{P} D_{t+1}\left(h_{t-1}\right): \quad U_{t}\left(h_{t-1}, z\right) \mathrm{d} \mu(z) \geq V_{t}\left(h_{t-1}\right) .
$$

Clearly, the fact that $\mathcal{P} D_{t+1}\left(h_{t-1}\right)$ is convex implies that $\mathcal{B} D_{t+1}\left(h_{t-1}\right)$ is convex.

Lemma $2 M_{t}: H_{t} \rightarrow \mathrm{R}$ and $V_{t}: H_{t-1} \rightarrow \mathrm{R}$ are lower semicontinuous. Moreover, $\mathcal{B} D_{t+1}: H_{t-1}{ }^{3} \quad \Delta\left(\Gamma_{t}(\cdot)\right)$ is an upper hemicontinuous correspondence with non-empty values that are convex and compact.

The proof of this makes use of Lemma 1 and is otherwise standard.

\subsection{The Forward Program}

Recall that extended histories in $\boldsymbol{A}_{t}$ consist of physical histories $h_{t}$ in $H_{t}$ augmented with the probability distributions over past actions whose support contains $h_{t}$. For any correspondence $F$ defined on $H_{t}$, we say that a function $f$ defined on $\boldsymbol{A}_{t}$ is an extended Borel measurable selection from $F$ if $f$ is Borel measurable and $f\left(\boldsymbol{q}_{t}\right) \in$ $F\left(\operatorname{proj}_{H_{\mathrm{t}}} \boldsymbol{\rho}_{t}\right)$. Extended measurable selections exist if measurable selections exist.

Lemma 3 Suppose $d_{t}$ is an extended Borel measurable selection from $\mathcal{B} D_{t+1}$. Then there exists a Borel measurable function $\gamma_{t}: R_{t-1} \rightarrow \Delta\left(C_{t}\right)$ and an extended Borel measurable selection $d_{t+1}$ from $D_{t+1}$ such that:

(i) $\gamma_{t}$ is optimal for the date $t$ consumer given the continuation $d_{t+1}$;

(ii) $d_{t}\left(\cdot \mid \boldsymbol{\Phi}_{t-1}\right)={ }^{\mathrm{R}} d_{t+1}\left(\cdot \mid \boldsymbol{\Phi}_{t-1}, c_{t}, \gamma_{t}\left(\cdot \mid \boldsymbol{\Phi}_{t-1}\right)\right) \mathrm{d} \gamma_{t}\left(c_{t} \mid \boldsymbol{\Phi}_{t-1}\right)$. 
P roof. Throughout the proof, take $h_{t-1}=\operatorname{proj}_{H_{\mathrm{t}-1}} \boldsymbol{\ell}_{t-1}$. Let $\gamma_{t}\left(\cdot \mid \boldsymbol{\ell}_{t-1}\right)$ be the marginal of $d_{t}\left(\cdot \mid \boldsymbol{\ell}_{t-1}\right)$ over $\Delta\left(C_{t}\right)$. By Lemma 12 of Harris, Reny and Robson (1995) there exists a Borel measurable function $\nu: \boldsymbol{A}_{t-1} \times C_{t} \rightarrow \Delta\left(\Gamma_{t+1}\left(H_{t}\right)\right)$ such that:

$$
d_{t}\left(\cdot \mid \boldsymbol{\ell}_{t-1}\right)={ }^{\mathbf{Z}} \nu\left(\cdot \mid \boldsymbol{\ell}_{t-1}, c_{t}\right) \mathrm{d} \gamma_{t}\left(c_{t} \mid \boldsymbol{\ell}_{t-1}\right) .
$$

By definition of $\mathcal{P} D_{t+1}\left(h_{t-1}\right), \nu\left(\cdot \mid \boldsymbol{R}_{t-1}, c_{t}\right)$ is in $D_{t+1}\left(h_{t-1}, c_{t}\right)$ for $\gamma_{t}\left(\cdot \mid \boldsymbol{q}_{t-1}\right)$-almost every $c_{t}$. One can therefore modify it on a set of measure zero to obtain a selection from $D_{t+1}$. The Measurable Maximum Theorem (Aliprantis and Border (1999, Theorem 17.91)) guarantees that the correspondence $\mathcal{A} M_{t}: H_{t}{ }^{3} \quad \Delta\left(\Gamma_{t+1}(\cdot)\right)$ admits a Borel measurable selection $\lambda$. Define:

$$
d_{t+1}\left(\cdot \mid \boldsymbol{\ell}_{t-1}, c_{t}, \mu\right)=\begin{array}{ll}
\nu\left(\cdot \mid \boldsymbol{\ell}_{t-1}, c_{t}\right) & \text { if } \mu(\cdot)=\gamma_{t}\left(\cdot \mid \boldsymbol{\ell}_{t-1}\right) \\
\lambda\left(\cdot \mid h_{t-1}, c_{t}\right) & \text { if } \mu(\cdot) \neq \gamma_{t}\left(\cdot \mid \boldsymbol{\ell}_{t-1}\right)
\end{array} .
$$

This construction makes $d_{t+1}$ an extended measurable selection from $D_{t+1}$. The definition of $\mathcal{B} D_{t+1}$ implies that if the date- $t$ consumer randomizes according to $\gamma_{t}$, then his utility will be:

$$
u_{t}\left(h_{t-1}, z\right) \mathrm{d} d_{t}\left(z \mid \ell_{t-1}\right) \geq V_{t}\left(h_{t-1}\right) .
$$

Alternatively, a deviation $\mu$ yields:

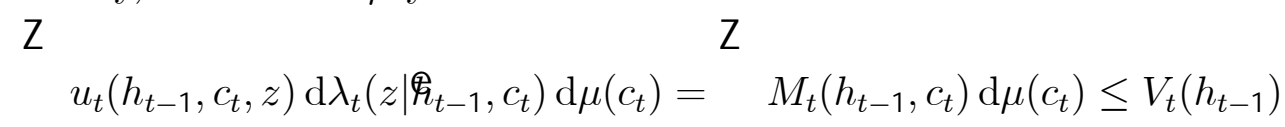

since $\lambda_{t}$ is a selection from $\mathcal{A} M_{t}$.

Notice that the observability of $\gamma_{t}\left(\cdot \mid \boldsymbol{\varphi}_{t-1}\right)$ is crucial for the construction of the continuation path faced by the date- $t$ consumer. Observability of mixed strategies also means that the date- $t$ consumer need not be indifferent between actions $c_{t} \in A_{t}\left(h_{t-1}\right)$ and associated continuation paths in $D_{t+1}\left(h_{t-1}, c_{t}\right)$ to be willing to randomize over these actions. Not randomizing may trigger a punishment in $\mathcal{A} M_{t}\left(h_{t-1}, c_{t}\right)$.

\subsection{Intrapersonal Equilibria}

The date- $T$ consumer faces a simple decision problem. For any $h_{T-1}$ in $H_{T-1}$, define:

$$
E_{T}\left(h_{T-1}\right)=\arg \max U_{T}^{1 / 2 Z}\left(h_{T-1}, c\right) \mathrm{d} \mu(c): \mu \in \Delta\left(A_{T}\left(h_{T-1}\right)\right)^{3 / 4} .
$$

By the Maximum Theorem, this defines an upper hemicontinuous correspondence with non-empty, convex and compact values. Using this correspondence as the input 
for the first iteration of the backward program, we can define $\left\{E_{t}\right\}_{t=1}^{T-1}$ recursively via $E_{t}=\mathcal{B} E_{t+1}$.

Proposition 1 The correspondence $E_{1}: H_{0}{ }^{3} \quad \Delta\left(\Gamma_{1}(\cdot)\right)$ is upper hemicontinuous and has non-empty values that are convex and compact. For every $h_{0} \in H_{0}, E_{1}\left(h_{0}\right)$ is the set of intrapersonal equilibrium paths given initial condition $h_{0}$.

The proof of Proposition 1 relies on standard arguments, and is therefore omitted.

\section{Compet it ive Equil ibrium}

We consider an exchange economy with a single consumption good at every date $t=1, \ldots, T$. There are $I$ types of consumers, indexed by $i=1, \ldots, I$. For every type, there is a continuum of consumers. For notational convenience we take this to be of unit measure for each type.

\subsection{Budget Sets and Preferences}

The set of initial conditions $H_{0}$ for the intrapersonal game of a consumer describes prices and initial endowments. Write $h_{0}=(p, e)$, where $p$ is a vector of prices and $e$ is a vector of endowments. We take $H_{0}$ to be some non-empty and closed subset of $\Delta_{T} \times \mathrm{R}_{++}^{T}$, where $\Delta_{T} \subset \mathrm{R}_{++}^{T}$ is the simplex of strictly positive prices for consumption at the $T$ dates. The date- $t$ consumer chooses a level of date- $t$ consumption subject to a budget constraint. Specifically, we take $C_{t}=\mathrm{R}_{+}$and define action correspondences as follows:

$$
\left.A_{t}\left(p, e, c_{1}, \ldots, c_{t-1}\right)=c_{t} \in \mathrm{R}_{+}:{ }_{s=1}^{X^{t}} p_{s} c_{s} \leq{ }_{s=1}^{X^{\Gamma}} p_{s} e_{s}\right)
$$

Since prices are strictly positive, it is clear that the action correspondence $A_{t}$ is continuous and compact-valued. Observe that for $t<T$ we do not let a date- $t$ consumer "throw away wealth:" what is not consumed is saved. ${ }^{3}$

The utility function for the date- $t$ consumer is defined over sequences of consumption choices $\left(c_{1}, \ldots, c_{T}\right) \in \mathbf{R}_{+}^{T}$. A consumer type is defined by an endowment vector $e_{i} \in \mathbf{R}_{++}^{T}$ and a vector of continuous utility functions $\left(U_{i, 1}, \ldots, U_{i, T}\right)$. Throughout, we maintain the following non-satiation assumption.

Assumption A For all $i$ and $t$, the utility function $U_{i, t}$ is weakly increasing in consumption at all dates, and strictly increasing in $c_{t}, \ldots, c_{T}$.

\footnotetext{
${ }^{3}$ In infinite-horizon versions of this type of intrapersonal game, it is possible to construct examples of (pure strategy) intrapersonal equilibria in which consumers throw away current consumption even though the preferences of every date-t consumer are strictly increasing in current and future consumption.
} 
This assumption does not cover models of habit persistence in which past consumption has a negative impact on current utility.

\subsection{The Excess Demand Correspondence}

Under the above assumptions, the intrapersonal game for a consumer of type $i$ has an intrapersonal equilibrium. We write $E_{i, 1}(p)=E_{1}\left(p, e_{i}\right)$ for the set of intrapersonal equilibrium paths for a consumer of type $i$ facing prices $p$. Proposition 1 ensures that $E_{i, 1}$ is upper hemicontinuous with non-empty convex and compact values.

Suppose that consumers of type $i$ all follow strategies that implement the path $\mathbb{R}_{i}(\cdot \mid p) \in E_{i, 1}(p)$. The aggregate consumption vector of consumers of type $i$ is then $c \mathrm{~d} \mu_{i}(c \mid p)$. Under these symmetric strategies, the excess demand correspondence of the type- $i$ consumers is given by:

$$
\zeta_{i}(p)={ }^{1 / 2 Z} c \mathrm{~d} \mu_{i}(c \mid p)-e_{i}: \mu_{i}(\cdot \mid p) \in E_{i, 1}(p)^{3 / 4} .
$$

The implied aggregate excess demand correspondence is:

$$
\zeta(p)={ }_{i=1}^{\left(\mathbf{X}^{I} \mathbf{Z}\right.}\left(c-e_{i}\right) \mathrm{d} \mu_{i}(c \mid p): \mu_{i}(\cdot \mid p) \in E_{i, 1}(p) \text { for all } i \text {. }
$$

For each $i$ the excess demand correspondence $\zeta_{i}$ is bounded below by $-e_{i}$. It is convex-valued since $E_{i, 1}$ is convex-valued for each $i$. Moreover, $\zeta_{i}(p)$ is the image of $E_{i, 1}(p)$ under the continuous mapping $\mu \mapsto c \mathrm{~d} \mu(c)-e_{i}$. The fact that $E_{i, 1}$ is upper hemicontinuous therefore implies that $\zeta_{i}$ is upper hemicontinuous.

The strict monotonicity of the preferences of the date- $T$ consumer implies that $p \cdot c=p \cdot e_{i}$ for all $c$ in the support of some path $\mu_{i}(\cdot \mid p) \in E_{i, 1}(p)$. Hence, for any $z \in \zeta(p):$

$$
p \cdot z=\mathrm{X}_{i=1}^{I} \mathbf{Z} p \cdot\left(c-e_{i}\right) \mathrm{d} \mu_{i}(c \mid p)=0 .
$$

Thus $\zeta$ satisfies Walras' law.

\subsection{The Boundary Behavior of Demand}

Our proof of existence of a competitive equilibrium is based on a theorem in Debreu (1982). For this to apply, we need to check that $\zeta$ satisfies the following boundary condition: if a sequence $\left\{\beta^{n}\right\}$ in $\Delta_{T}$ converges to $p \in \partial \Delta_{T}$, then $\inf _{z \in \zeta\left(p^{n}\right)}\|z\|$ goes to infinity, where $\|z\|={ }_{t=1}^{T}\left|z_{t}\right|$. One way to obtain this boundary condition is the following assumption on preferences. Observe that the assumption implies that utility must be bounded below.

A ssumption B For every $t=1, \ldots, T$, the preferences of the date $t$ consumer can 
be represented as:

$$
U_{i, t}\left(c_{1}, \ldots, c_{T}\right)={ }_{n=0}^{\mathbb{X}^{-t}} \delta_{i, n} u_{i}\left(c_{t+n}\right),
$$

where $u_{i}: \mathbf{R}_{+} \rightarrow \mathbf{R}$ is continuous, concave, strictly increasing and unbounded above. The subjective discount factors $\delta_{i, 1}, \ldots, \delta_{i, T}$ are strictly positive.

This assumption covers the preferences proposed by Strotz (1956) and Phelps and Pollak (1968) and used more recently by Laibson (1997) and others. The preferences of consumers of type $i$ over future consumption sequences change over time whenever $\delta_{i, t}$ is not an exponential function of $t$.

Lemma 4 Suppose that $\left(U_{i, 1}, \ldots, U_{i, T}\right)$ satisfies A ssumption B. If a sequence $\left\{p^{n}\right\}$ in $\Delta_{T}$ converges to a price in $\partial \Delta_{T}$, then $\inf _{z \in \zeta_{\mathrm{i}}\left(p^{\mathrm{n}}\right)}\|z\|$ goes to $+\infty$.

P roof. The proof is by induction on the length of the game. Take $T=2$. Note that the date-1 consumer faces a standard decision problem. The result is then a direct consequence of Debreu (1982, Lemma 4) since the preferences of the date-1 consumer are strictly increasing. Note in addition that the expected utility of the date- 1 consumer goes to $+\infty$ as $\left\{p^{n}\right\}$ goes to $p \in \partial \Delta_{T}$. Now suppose that the result is true for any game of length $T$ and suppose further that in any such game expected utility of the date- 1 consumer goes to $+\infty$ uniformly across intrapersonal equilibria as $\left\{p^{n}\right\}$ converges to $p \in \partial \Delta_{T}$. We want to show that the same is true for a game of length $T+1$.

Suppose first that $\left\{p_{1}^{n}\right\}$ goes to zero. Then the date- 1 consumer can guarantee for himself a utility of $+\infty$ in the limit by spending all his wealth on current consumption, irrespective of the behavior of his successors. This implies that the expected utility of the date- 1 consumer must go to $+\infty$ uniformly across equilibria. Formally, for all sequences $\left\{\mu_{i}^{n}\right\}$ that satisfy $\mu_{i}^{n} \in E_{i, 1}\left(p^{n}\right)$ :

$$
\lim _{n \rightarrow \infty}{ }^{Z} U_{i, 1}(c) \mathrm{d} \mu_{i}^{n}(c)=+\infty .
$$

The concavity of $u_{i}$ together with Jensen's inequality implies:

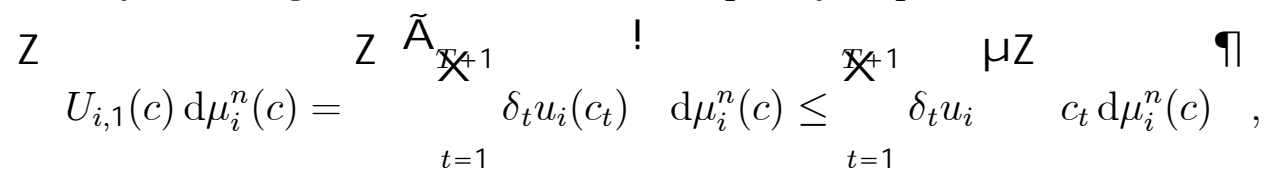

and from this it then follows that:

$$
\lim _{n \rightarrow \infty} \mathrm{X}_{t=1}^{+1} \mathbf{Z}
$$

This implies the desired boundary behavior. 
Suppose alternatively that $\left\{p_{1}^{n}\right\}$ is bounded away from zero and some other price

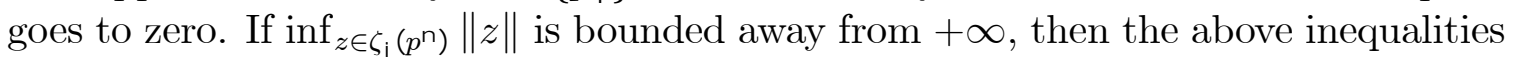
imply that there must be a sequence of intrapersonal equilibria along which expected date-1 utility remains bounded. But this cannot be part of an optimal strategy for the date-1 consumer. To see this, suppose the date- 1 consumer leaves nominal wealth $\varepsilon>0$ to the date- 2 consumer. Then the induction hypothesis would imply that the utility of the date- 2 consumer would go to $+\infty$ uniformly across intrapersonal equilibria along the considered price sequence. More specifically:

$$
\lim _{n \rightarrow \infty} \max _{t \in\{2, \ldots, T+1\}} u_{i}\left(c_{t}\right) \mathrm{d} \mu_{i, \varepsilon}^{n}(c)=+\infty
$$

uniformly across equilibrium continuation paths $\mu_{i, \varepsilon}^{n} \in \Delta\left(\Gamma_{2}\left(p^{n}, e_{i},\left(p^{n} e_{i}-\varepsilon\right) / p_{1}^{n}\right)\right)$ that follow when the date- 1 consumer leaves nominal wealth $\varepsilon>0$ to the date- 2 consumer. This implies that the date- 1 consumer could guarantee for himself infinite utility in the limit, a contradiction.

We can now apply Debreu (1982, Theorem 8) to obtain the following result.

P roposition 2 Suppose that $\left(U_{i, 1}, \ldots, U_{i, T}\right)$ satisfies Assumption B for at least one $i$. Then there exists a competitive equilibrium in which consumers of the same type follow the same strategies.

The arguments given in the proof of Lemma 4 do not rely on the convexity of the individual excess demand correspondences. An analogue of Proposition 2 can therefore be proved for intrapersonal equilibria in pure strategies. The aggregate demand correspondence for consumers of a given type is made convex by assigning different strategies to different consumers of the same type. As pointed out earlier, these pure-strategy intrapersonal equilibria exist by the results of Harris (1985).

We use the convexity of the individual demand correspondences to construct a symmetric competitive equilibrium. In economies in which preferences do not change, situations in which the demand correspondence is not a singleton arise only when a consumer is indifferent between several choices. Convexity of preferences then implies that the demand correspondence must have convex values. Here, it is typically not true that the date- $t$ consumer is indifferent between the various elements of the subgame-perfect equilibrium correspondence at time $t$.

\section{Concluding Remarks}

We have used certain assumptions about preferences to guarantee that a competitive equilibrium exists when preferences change over time. Our assumptions about preferences are no doubt more restrictive than needed. Further research is required to determine the extent to which they can be weakened. 
Equilibria that are symmetric in the sense that consumers of the same type follow the same strategies exist if consumers can make their current behavior depend on past randomized behavior. This ensures convexity of the correspondence of intrapersonal equilibria. We use this convexity as part of a classic existence proof along the lines of Debreu (1982). It may be the case that the intrapersonal equilibrium correspondence has sufficient regularity to allow us to do without this convexity. We could then show the existence of a competitive equilibrium without having to assume that there is a continuum of consumers of every type.

\section{Ref er ences}

[1] Aliprantis, C.D. and K.C. Border (1999): Infinite Dimensional Analysis: A Hitchhiker's Guide, Second Edition, Springer Verlag.

[2] Debreu, G. (1982): "Existence of Competitive Equilibrium," in Handbook of M athematical Economics, Volume II, Elsevier North-Holland.

[3] Goldman, S. (1980): "Consistent Plans," Review of Economic Studies, Vol. 47, No. 3 (April), 533-537.

[4] Harris, C. (1985): "Existence and Characterization of Perfect Equilibrium in Games of Perfect Information," Econometrica, Vol. 53, No. 3 (May), 613-628.

[5] Harris, C., and D. Laibson (2001): "Dynamic Choices of Hyperbolic Consumers," E conometrica, Vol. 69, No. 4 (July), 935-957.

[6] Harris, C., P. Reny and A. Robson (1995): "The Existence of Subgame-Perfect Equilibrium in Continuous Games with Almost Perfect Information: A Case for Public Randomization," Econometrica, Vol. 63, No. 3 (May), 507-544.

[7] Laibson, D. (1997): "Golden Eggs and Hyperbolic Discounting," Quarterly J ournal of Economics, Vol. CXII, No. 2 (May), 443-477.

[8] Luttmer, E.G.J. and T. Mariotti (2000): "Subjective Discounting in an Exchange Economy," working paper.

[9] Luttmer, E.G.J. and T. Mariotti (2002): "Efficiency and Equilibrium when Preferences are Time-Inconsistent," working paper.

[10] Mariotti, T. (2000): "Subgame-Perfect Equilibrium Outcomes in Continuous Games of Almost Perfect Information," J ournal of M athematical E conomics, 34, 99-128.

[11] Peleg, B., and M. Yaari (1973): "On the Existence of a Consistent Course of Actions when Tastes are Changing," Review of Economic Studies, Vol.40, No. 3 (July), 391-401. 
[12] Phelps, E.S., and R.A. Pollak (1968): "On Second-Best National Saving and Game-Equilibrium Growth," Review of Economic Studies, Vol. 35, No. 2 (April), 185-199.

[13] Pollak, R.A. (1968): "Consistent Planning," Review of Economic Studies, Vol. 35, No. 2 (April), 201-208.

[14] Simon, L.K. and W.R. Zame (1990): "Discontinuous Games and Endogenous Sharing Rules," Econometrica, Vol. 58, No. 4 (July), 861-872.

[15] Strotz, R.H. (1956): "Myopia and Inconsistency in Dynamic Utility Maximization," Review of Economic Studies, Vol. 23, No. 3, 165-180. 